\title{
Photometric Recovery of Ortho-images Derived from Apollo 15 Metric Camera Imagery
}

\author{
Taemin Kim ${ }^{1}$, Ara V. Nefian², and Michael J. Broxton ${ }^{1,2}$ \\ 1. NASA Ames Research Center, Moffett Field, CA, 94035 \\ 2. Carnegie Mellon University
}

\begin{abstract}
The topographical and photometric reconstruction of the moon from Apollo metric data has gained attention to support manned mission planning since the NASA has been working on return to the moon in 2004. This paper focuses on photometric recovery of the moon surface from Apollo orbital imagery. The statistical behavior of photons generates the scene radiance which follows a continuous Poisson distribution with the mean of surface radiance. The pixel value is determined by the camera response of sensor exposure which is proportional to scene radiance and exposure time. The surface radiance, exposure time and camera response are estimated by the maximum likelihood method for sensor exposure. The likelihood function is highly nonlinear and we were unable to find an estimator in closed form. Grouping the three sets of parameters (surface radiance, exposure time, and camera response), an EM-like juggling algorithm is proposed to determine the one family of parameters from the others. The photometric recovery of otho-images derived from Apollo 15 metric camera imagery was presented to show the validity of the proposed method.
\end{abstract}

\section{Introduction}

The Lunar Mapping Modeling Project (LMMP) has been actively carried out to develop maps and tools to benefit the Constellation Program (CxP) lunar planning. It will provide common, consistent and useful access to this information for lunar exploration and science communities. One of the requirements for LMMP is to construct geo-registered global and local albedo (visible image) base maps of the Moon from the digital stereo pair scans collected by Apollo era lunar missions (Figure 1). These scans, despite their high quality, are affected by noise inherent to the scanning process: the presence of film grain, dust and lint particles. Attenuating the effect of these scanning artifacts and estimating the surface radiance from Apollo orbital imagery are the central focus of this paper.

More than ever, scanned images are used as texture maps for geometric models. When a picture of a scene is taken and digitized to obtain "brightness" values, these values are rarely true measurements of relative radiance in the scene. There is usually a nonlinear 


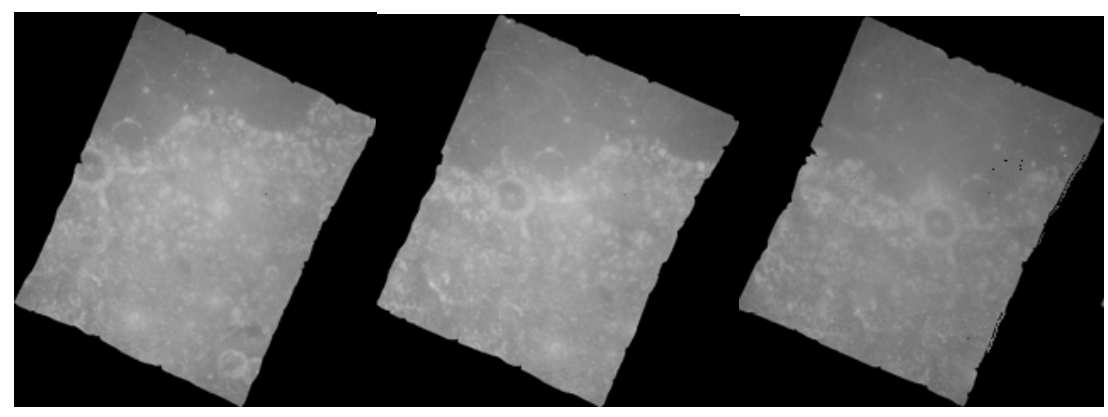

Figure 1: Examples of Ortho-images from Apollo 15 Metric Camera Imagery.

mapping that determines how radiance in the scene becomes pixel values in the image [1]. The image acquisition pipeline shows how the nonlinear mapping composite the each component in a digital image formation (Figure 2).

In this paper, the scene radiance is modeled as a continuous Poisson distribution with surface radiance due to the statistical behavior of photons. The pixel value is determined by the camera response of sensor exposure which is proportional to scene radiance and exposure time. The surface radiance, exposure time and camera response are estimated by the maximum likelihood method for sensor exposure. The likelihood function of all parameters is highly nonlinear and an estimator was unable to be found in in closed form. An EM-like juggling algorithm is proposed to determine the one family of parameters from the others. Finally, the reconstructed radiance map from lunar orbital imagery is presented.

\section{Image formation}

Scene radiance becomes pixel values through several linear and nonlinear transformations as seen in the image acquisition pipeline (Figure 2). These unknown nonlinear mapping scan occur during exposure, development, scanning, digitization, and remapping. The camera response function is the aggregate mapping from sensor exposure $X$ to pixel values $Z$. We estimate it from a set of sufficiently overlapped images with different exposure times, as described in [1].

After the development, scanning and digitization processes, we obtain an intensity value $Z$, which is a nonlinear function of the original exposure $X$ at the pixel. Let us call this function $f$, which is the composition of the characteristic curve of the film as well as all the nonlinearities introduced by the later processing steps. We write down the film reciprocity equation as:

$$
Z=f(X) .
$$

Since we assume $f$ is monotonic, it is invertible, and we can rewrite (1) as: 


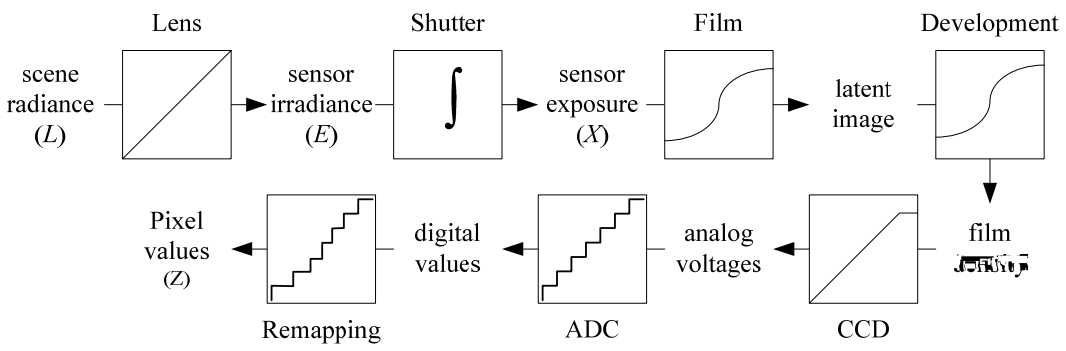

Figure 2: Image Acquisition Pipeline

$$
X=g(Z) .
$$

where $g=f^{-1}$.

A continuous Poisson distribution is adopted to model the scene radiance and sensor exposure. Several sources of image noise are listed in [2], but the photon noise dominates the other components in CCD or CMOS cameras. The other noise can be reduced by appropriate design of manufacturer and negligible. Photon production is governed by the laws of quantum physics which restrict us to consider an average number of photons within a give observation window. The probability distribution of $p$ photons during seconds is known to be discrete Poisson [2,3]:

$$
P(p \mid \rho, t)=\frac{(\rho t)^{p} e^{-\rho t}}{p !},
$$

where $\rho$ is the rate or intensity parameter measured in photons per second. By the continuous nature of measurement, the sensor exposure $X$ is represented by the continuous Poisson distribution:

$$
P(x \mid r, t)=\frac{(r t)^{x} e^{-r t}}{\Gamma(x+1)},
$$

where $r$ is the scene radiance.

\section{Radiance Maps from Apollo Imagery}

The input to our algorithm is $n$ digitized photographs taken from the same vantage point with different known exposure durations $t_{j}(j=1,2, \cdots, n)$. Let the pixel values be uniformly quantized. In this case, 256 gray levels $(z=0,1, \cdots, 255)$. We will assume that the scene is static and that lighting changes can be safely ignored. For brevity and 


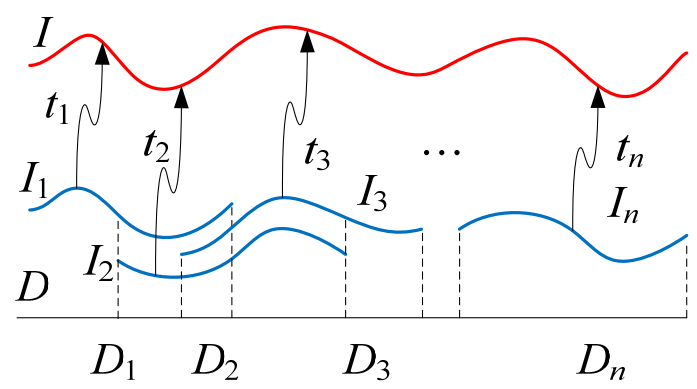

Figure 3: Radiance Map Recovered from Orbital Imagery.

simplicity, one-dimensional illustrations of images will be presented which can be easily extended to two-dimensional images (Figure 3).

Suppose that we have perfectly aligned images on a regular grid (Figure 4). It can then be assumed that each pixel value on a grid point comes from the same radiance value on that point. We denote $i$ by a spatial index over pixels and $j$ by an image index. Let $r_{i}$ be the radiance value on $i$ th grid point and $t_{j}$ be the exposure time of $j$ th image. The inverse function of camera response is represented by a vector. We will denote sensor exposure and pixel values by $x_{i j}$ and $z_{i j}$, respectively. From (2) we can write

$$
x_{i j}=g\left(z_{i j}\right) \text {. }
$$

All parameters such as surface radiance, exposure time, and camera response are estimated by the maximum likelihood method of the continuous Poisson distribution. Let $\boldsymbol{g}, \boldsymbol{r}$, and $\boldsymbol{t}$ be the parameterized vectors for camera response, sensor irradiance and exposure time, respectively. We then have their likelihood of the continuous Poisson distribution:

$$
\begin{aligned}
l(\boldsymbol{g}, \boldsymbol{r}, \boldsymbol{t}) & =\prod_{j=1}^{n} \prod_{i \in D_{j}} p\left(g\left(z_{i j}\right) \mid r_{i} t_{j}\right) \\
& =\prod_{j=1}^{n} \prod_{i \in D_{j}} \frac{\left(r_{i} t_{j}\right)^{g\left(z_{i j}\right)} e^{-r_{i} t_{j}}}{\Gamma\left(g\left(z_{i j}\right)+1\right)} .
\end{aligned}
$$

subject to

$$
0 \leq g(0) \leq g(1) \leq \cdots \leq g(254) \leq g(255) .
$$

Taking the natural logarithm of (6), we have:

$$
\begin{aligned}
L(\boldsymbol{g}, \boldsymbol{r}, \boldsymbol{t}) & =\ln l(\boldsymbol{g}, \boldsymbol{r}, \boldsymbol{t}) \\
& =\sum_{j=1}^{n} \sum_{i \in D_{j}}\left\{g\left(z_{i j}\right) \ln r_{i} t_{j}-r_{i} t_{j}-\ln \Gamma\left(g\left(z_{i j}\right)+1\right)\right\} .
\end{aligned}
$$




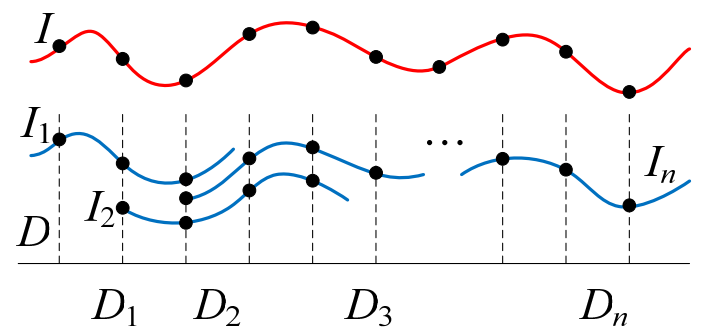

Figure 4: Aligned Image Set in Regular Grid.

Taking the derivative with respect to $r_{i}, t_{j}$ and $g(k)$, we have:

$$
\begin{aligned}
\frac{\partial L}{\partial r_{i}} & =\sum_{i \in D_{j}}\left\{\frac{g\left(z_{i j}\right)}{r_{i}}-t_{j}\right\}, \\
\frac{\partial L}{\partial t_{j}} & =\sum_{i \in D_{j}}\left\{\frac{g\left(z_{i j}\right)}{t_{j}}-r_{i}\right\}, \\
\frac{\partial L}{\partial g(k)} & =\sum_{k=z_{i j}}\left\{\ln r_{i} t_{j}-\Psi(g(k)+1)\right\},
\end{aligned}
$$

where $\Psi$ is the digamma function.

\section{Juggling Algorithm}

Since we were unable to find a close form solution to make (9) zeros, we determine them iteratively as in the expectation maximization (EM) method. Fortunately, we have the closed-form solution for scene radiance and exposure times from (9):

$$
\begin{gathered}
r_{i}=\frac{\sum_{i \in D_{j}} g\left(z_{i j}\right)}{\sum_{i \in D_{j}} t_{j}}, \\
t_{j}=\frac{\sum_{i \in D_{j}} g\left(z_{i j}\right)}{\sum_{i \in D_{j}} r_{i}},
\end{gathered}
$$

We have the closed-form solution for the camera response function from (9) as long as it satisfies the increasing property (7): 


$$
g(k)=\Psi^{-1}\left(\frac{\sum_{k=z_{i j}} \ln r_{i} t_{j}}{\sum_{k=z_{i j}} 1}\right)-1 .
$$

In practice, we have to optimize (8) directly with linear constraints of (7). This optimization is stable in that the objective function and domain are convex. Still, (12) is useful to provide a good initial guess of the optimization. The following approximation is also useful unless we have a built-in function code of the inverse digamma function:

$$
g(k)=\exp \left(\frac{\sum_{k=z_{i j}} \ln r_{i} t_{j}}{\sum_{k=z_{i j}} 1}\right) .
$$

This is based on the fact that the digamma function has the same value with the logarithm function asymptotically:

$$
\Psi(x+1) \simeq \ln x .
$$

Iteratively we can update all parameters from an initial guess to the convergence. We call it juggling algorithm as the one family of parameters are fully determined by the others. It is reasonable to choose exposure times uniformly because the images were taken continuously. The camera response function is initialized linearly because most cases it follows the gamma correction function.

The extension to the case of the unaligned images is straightforward for the exposure times and the camera response function in that they have sufficient number of real observations to be determined. However, scene radiance should be determined by a single point of interest because there is no corresponding observation in the other overlapping images. This eliminates the robustness of the estimation. To avoid single observations, virtual observations for all real observations are generated in all other images. Figure 5 illustrates that the virtual observations (hollow points) are interpolated bilinearly to real observations (solid points). But, it is sufficient to calculate exposure times and camera response function from the real observations as in the regular case. The virtual observations

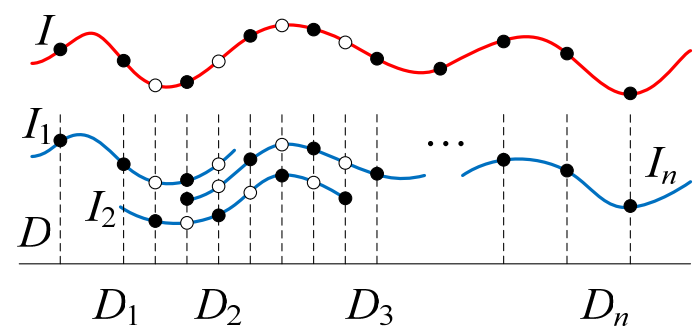

Figure 5: Unaligned Image Set with Virtual Pixels. 
are used to calculate the radiance:

$$
r_{i}=\frac{\sum_{i \in D_{j}} \tilde{g}\left(z_{i j}\right)}{\sum_{i \in D_{j}} t_{j}},
$$

where $\tilde{g}\left(z_{i j}\right)$ is the estimated sensor exposure for real observation or interpolated values for virtual observations.

\section{Experimental Results}

The National Aeronautics and Space Administration (NASA) Exploration Systems Mission Directorate (ESMD) has been charged with producing cartographic products via LMMP for use by mission planners and scientists in NASA's Constellation program. As part of the

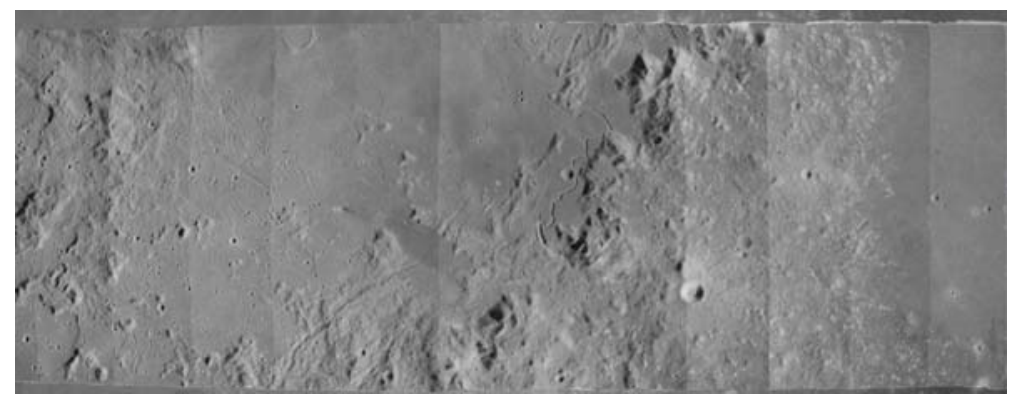

(a) Original Image Mosaic

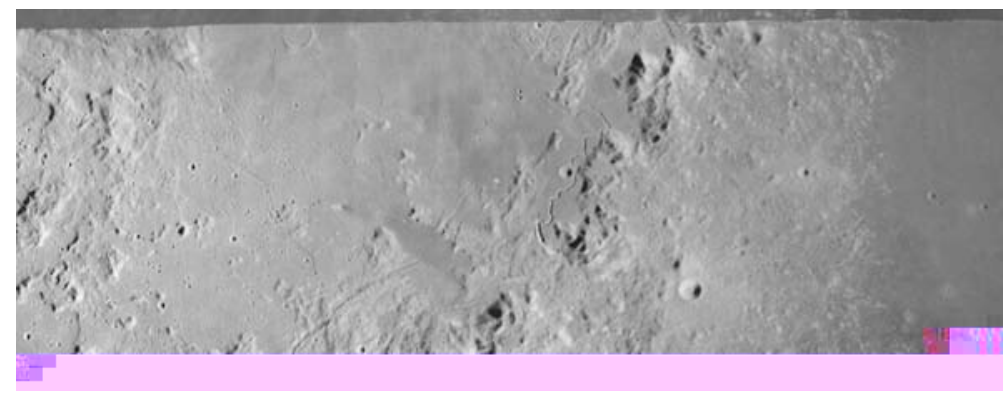

(b) Photometric Recovery Map

Figure 6: Radiance Maps of Subset Images. 
LMMP, we have produced 70 preliminary Digital Terrain Models (DTMs) and ortho-images derived from Apollo 15 Metric Camera (AMC) orbit 33 imagery using the Ames Stereo Pipeline (ASP); a software tool that generates high quality DTMs from orbital imagery using a fully automated process. Given a pair in Apollo Metric Imagery, the reference image is projected onto the reconstructed DTM from the pair by ASP and then the ortho-image is reconstructed by orthographic projection. The whole image set consists of 66 ortho-images and has significant overlap between adjacent frames (80\%) so that it is well-suited for photometric recovery.

The photometric recovery program is implemented based on the NASA Vision Workbench (VW). The NASA VW is a general purpose image processing and computer vision library developed by the Intelligent Robotics Group in the Intelligent Systems Division at the NASA Ames Research Center. Figure 6 shows the original mosaic image constructed from ortho-images and photometrical radiance maps from the consecutive 12 images. The relative radiance is adjusted to be consistent with the remaining images. As you can see in the figure, the original image mosaic shows the vertical seams on the overlapping boundaries. However, the proposed method provides the seamless radiance map. Figure 7 shows the whole image mosaics of original images and the proposed method. The aspect ratio is adjusted because of limited space.

The camera response is shown in Figure 8. The initial guess by (13) is much closer to the optimal solution. The camera response function is obtained by the inverse function shown in Figure 8b. The estimated exposure times of each image are shown in Figure 9.

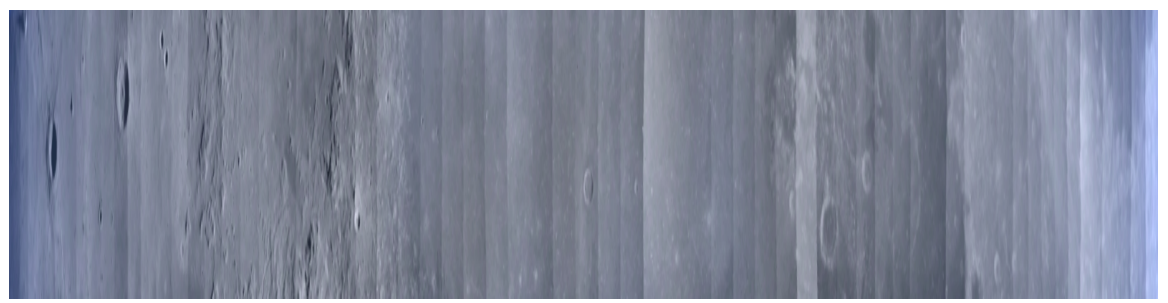

(a) Original Image Mosaic

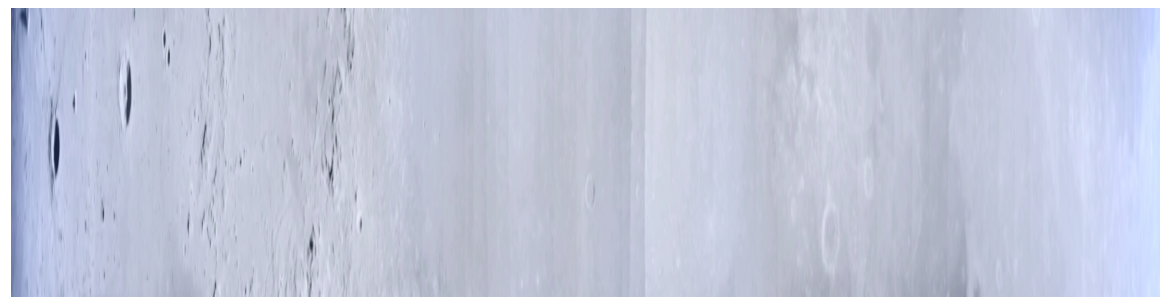

(b) Photometric Recovery Map

Figure 7: Radiance Maps of Full Set. 


\section{Conclusion}

The photometric radiance map of the moon was successfully reconstructed from Apollo 15 metric camera imagery. The pixel value is determined by the camera response of sensor exposure which is proportional to scene radiance and exposure time. The statistical

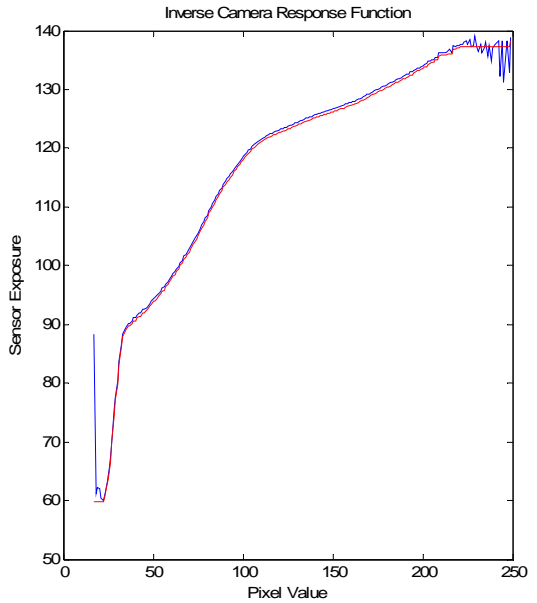

(a) Inverse Camera Response

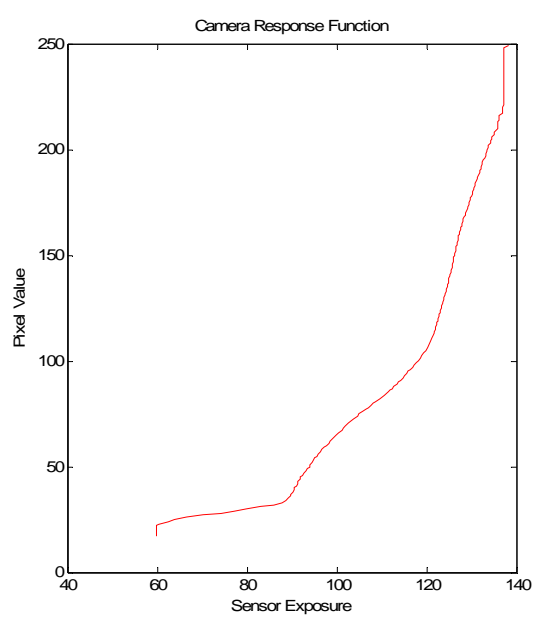

(b) Camera Response Function

Figure 8: Camera Response Functions.

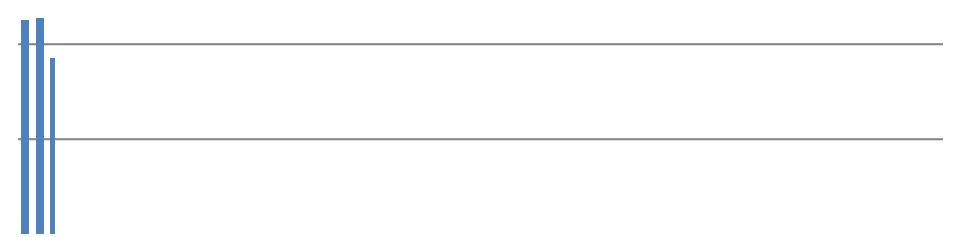


behavior of photons was considered and the maximum likelihood function of the parameters was derived. The surface radiance, exposure time and camera response are estimated by the maximum likelihood method. The likelihood function is highly nonlinear so that the three sets of parameters (surface radiance, exposure time, and camera response) are iteratively optimized. A juggling algorithm is proposed to determine the one family of parameters from the others. The experimental results show the validity of the proposed method.

A residual analysis would be desirable to provide a quantitative measure of the proposed method. A parametric representation of surface radiance would also be valuable to enhance the recovery resolution and robustness of the algorithm.

\section{Acknowledgements}

This work was funded by the NASA Lunar Advanced Science and Exploration Research (LASER) program grant \#07-LASER07-0148, NASA Advanced Information Systems Research (AISR) program grant \#06-AISRP06-0142, and by the NASA ESMD LMMP. The first author conducted the research under the Visiting Researcher Agreement between the NASA and the Korea Advanced Institute of Science and Technology.

\section{References}

1. Paul E. Debevec and Jitendra Malik, Recovering high dynamic range radiance maps from photographs. In SIGGRAPH 97 Conference Proceedings, Addison Wesley, T. Whitted, Ed., Annual Conference Series, ACM SIGGRAPH, 369-378, 1997.

2. I. Young, J. Gerbrands, and L. van Vliet. Fundamentals of image processing, 1995. Delft University of Technology.

3. Youngbae Hwang, Jun-Sik Kim and In-So Kweon, "Sensor noise modeling using the Skellam distribution: Application to the color edge detection", in IEEE International Conference on Computer Vision and Pattern Recognition (CVPR), 2007.

4. S. J. Lawrence, M. S. Robinson, M. Broxton, J. D. Stopar, W. Close, J. Grunsfeld, R. Ingram, L. Jefferson, S. Locke, R. Mitchell, T. Scarsella, M. White, M. A. Hager, T. R. Watters, E. Bowman-Cisneros, J. Danton, and J. Garvin. The Apollo Digital Image Archive: New Research and Data Products. In Proc of the NLSI Lunar Science Conference, page 2066, 2008.

5. M. J. Broxton, Z. M. Moratto, A. Nefian, M. Bunte, M. S. Robinson, Preliminary Stereo Reconstruction from Apollo 15 Metric Camera Imagery, 40th Lunar and Planetary Science Conference (2009)

6. W. Triggs, P. McLauchlan, R. Hartley, and A. Fitzgibbon. Bundle Adjustment: A Modern Synthesis. In Vision Algorithms: Theory and Practice, number 1883 in LNCS, pages 298-373. Springer-Verlag, Corfu, Greece, September 1999. 